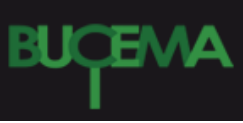

Bulletin du centre d'études médiévales d'Auxerre | BUCEMA

Hors-série $n^{\circ} 6$ | 2013

Autour du cloître : les chapelles Notre-Dame et les accès au chapitre

\title{
Une chapelle mariale romane à l'abbaye de Baume- les-Messieurs (Jura) ? Nouvelles données archéologiques du bâti
}

Sébastien Bully et Marie-Laure Bassi

\section{(2) OpenEdition}

Édition électronique

URL : https://journals.openedition.org/cem/12665

DOI : $10.4000 /$ cem. 12665

ISSN : 1954-3093

Éditeur

Centre d'études médiévales Saint-Germain d'Auxerre

Référence électronique

Sébastien Bully et Marie-Laure Bassi, « Une chapelle mariale romane à l'abbaye de Baume-les-

Messieurs (Jura) ? Nouvelles données archéologiques du bâti », Bulletin du centre d'études médiévales d'Auxerre / BUCEMA [En ligne], Hors-série $n^{\circ} 6$ | 2013, mis en ligne le 17 avril 2013, consulté le 03 mars 2023. URL : http://journals.openedition.org/cem/12665 ; DOI : https://doi.org/10.4000/cem.12665

Ce document a été généré automatiquement le 3 mars 2023

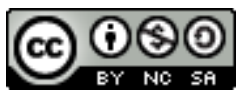

Creative Commons - Attribution - Pas d'Utilisation Commerciale - Partage dans les Mêmes Conditions 4.0 International - CC BY-NC-SA 4.0

https://creativecommons.org/licenses/by-nc-sa/4.0/ 


\title{
Une chapelle mariale romane à l'abbaye de Baume-les-Messieurs (Jura) ? Nouvelles données archéologiques du bâti
}

\author{
Sébastien Bully et Marie-Laure Bassi
}

1 L'abbaye de Baume-les-Messieurs est étroitement liée à l'histoire de Cluny, mais malgré cette filiation illustre, le monastère roman reste encore, pour une grande part, méconnu. Les recherches archéologiques menées sur l'abbatiale depuis 2007 ont largement enrichi nos connaissances sur l'édifice ${ }^{1}$, mais les autres bâtiments de l'abbaye n'ont encore

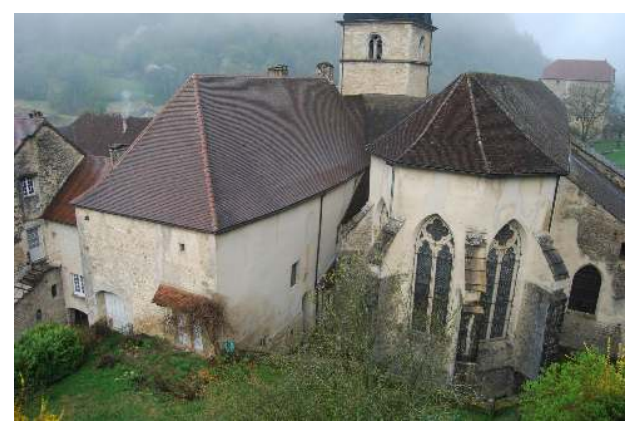
que très peu été étudiés en raison d'importantes transformations qui les ont affectés à la fin du Moyen Âge et à l'époque Moderne. L'abandon de la vie communautaire à la fin du XVII ${ }^{\mathrm{e}}$ siècle entraîna en effet des modifications inhérentes au nouveau mode de vie des religieux et une grande partie des bâtiments, en particulier ceux situés à l'est du cloître, fut transformé en demeures individuelles pour les chanoines, avant d'être vendus à des particuliers en 1791. Cette privatisation des bâtiments claustraux et leur parcellisation, qui perdurent de nos jours, ne facilitent pas l'accès à certains bâtiments pour leur étude.

2 Cependant, c'est dans la cave de l'une de ces maisons particulières que des vestiges pouvant appartenir à une chapelle mariale romane ont été récemment identifiés, à l'emplacement d'une chapelle de la fin du Moyen Âge connue par les textes ${ }^{2}$. 
Fig. 1 - Plan général de Baume-les-Messieurs, état actuel.

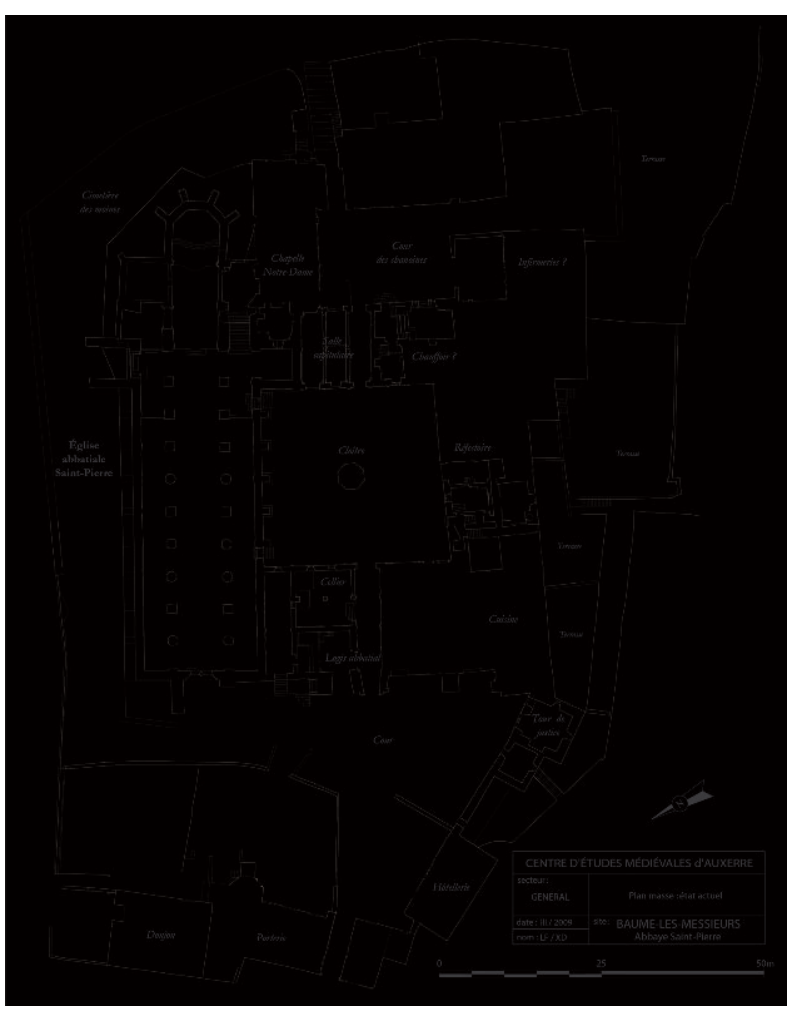

\section{Bilan historiographique}

3 L'existence dans le monastère de Baume d'une chapelle dédiée à la Vierge est attestée par les textes à partir du Xve siècle. Identifiée comme la chapelle Notre-Dame de Salins, son emplacement était inconnu. Dans son mémoire rédigé vers 1760, l'abbé de Broglie (abbé de Baume de 1714 à 1766) la tenait pour disparue ${ }^{3}$. D. Monnier situait la chapelle de Salins au nord de la première travée du chœur de l'église ${ }^{4}$ alors qu'A. Rousset soupçonnait, sans toutefois en préciser son emplacement, que cette chapelle «n'était point dans l'église abbatiale, ainsi que le dit M. Monnier " ${ }^{5}$. L.-A. Roy approchait de la vérité en écrivant qu'Henri de Salins "adossa au clocher la chapelle qu'on désigne encore par son nom ${ }^{6}{ }^{6}$. Enfin, en 1978, les auteurs de L'abbaye de Baume-les-Messieurs renoncèrent à la situer, estimant que "la localisation et la raison d'être de cette chapelle ne sont pas sans soulever de nombreux problèmes " ${ }^{7}$.

4 Il faut attendre les recherches de Sandrine Roser pour que les choses s'éclaircissent ${ }^{8}$. Cette dernière va identifier et situer la chapelle Notre-Dame de Salins grâce à une étude approfondie des archives modernes où des bribes d'information permettent de localiser l'emplacement de la chapelle de Salins dans la troisième cour de l'abbaye ${ }^{9}$, près de l'abbatiale, mais indépendante de celle-ci. Cette chapelle se situait donc au sud de l'abside et à l'est du bras sud du transept, à l'emplacement actuel d'une maison d'habitation. 
Fig. 2 - Baume-les-Messieurs, vue générale depuis le nord-est de l'ancienne chapelle de Salins transformée en maison (cl. S. Bully).

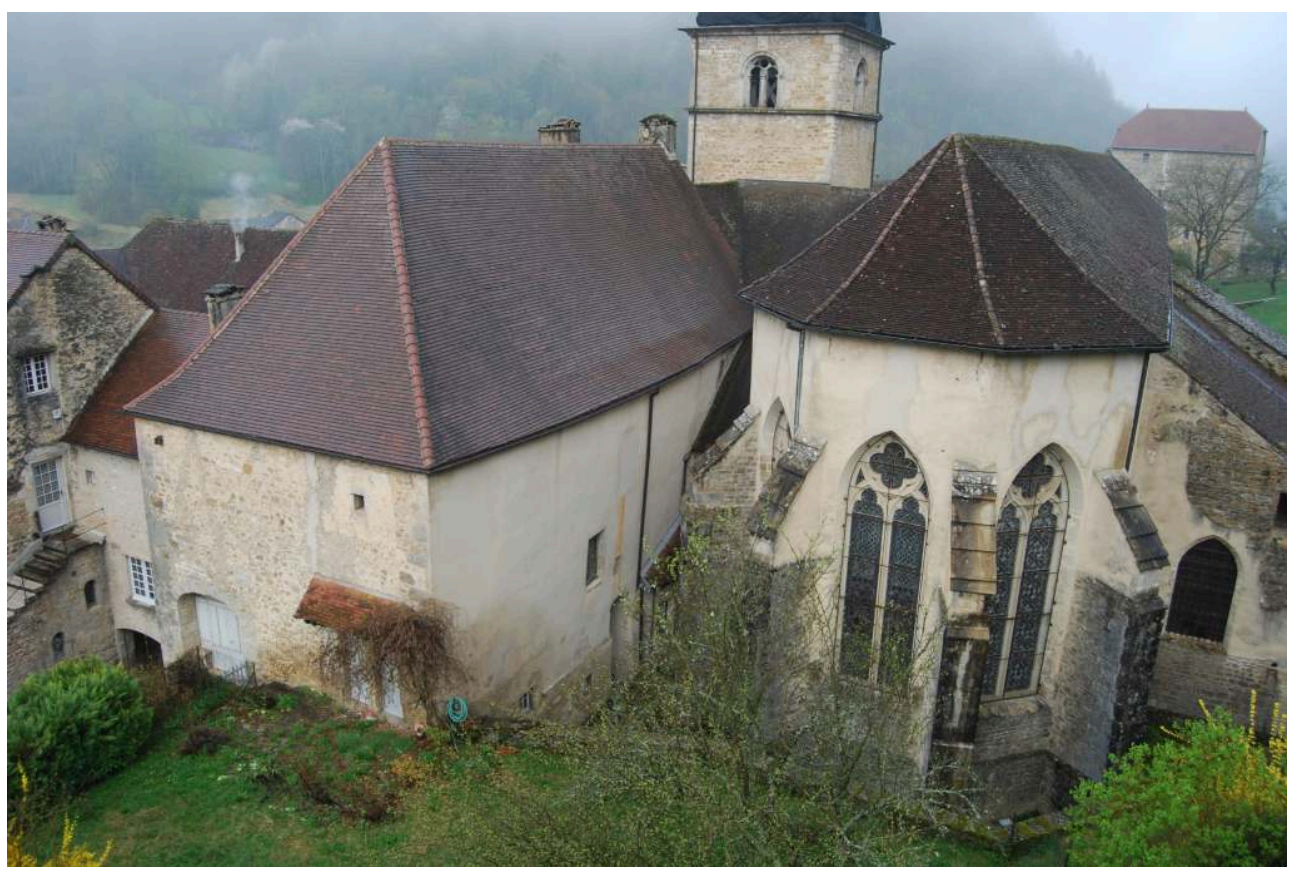

\section{La chapelle gothique Notre-Dame de Salins}

La chapelle apparaît le plus souvent dans la documentation sous le nom de chapelle «de Salins » à cause du nom de son fondateur, l'abbé Henri de Salins. Son acte de fondation du 20 août 1452 nous renseigne essentiellement sur la présentation et les modalités de financement des différentes messes devant y être célébrées, mais n'apporte que peu de renseignements sur l'édifice lui-même. Le document évoque clairement, mais brièvement, la construction de la chapelle dans laquelle Henri de Salins se fit inhumer. Elle a dû être élevée durant son abbatiat, c'est-à-dire entre 1432 et le premier semestre 1452 .

L'acte de fondation de 1452 montre sans équivoque que la chapelle était contiguë à l'église abbatiale. Vingt ans plus tard, un autre document rappelle le voisinage des deux édifices ${ }^{10}$ et c'est en 1685 que la chapelle Notre-Dame perdit l'aspect d'un édifice religieux, et rapidement, le souvenir même de son emplacement exact, voire de son existence, tomba dans l'oubli. La chapelle de Salins fut transformée en maison d'habitation, car elle était alors qualifiée d'«innuttille, séparée de l'église depuis longtemps, pollué, et de tout temps sans fondation $"{ }^{11}$.

7 La largeur de la maison correspond vraisemblablement à celle de l'ancienne chapelle gothique dont les fondations et peut-être une partie des élévations sont conservées. D'ailleurs, l'acte de 1685 ne prévoyait pas expressément une augmentation de la surface habitable offerte par la chapelle. En l'état actuel, les sources d'archives comme archéologiques ne permettent pas de restituer précisément l'élévation de cet édifice, mais étant donné la faible largeur de la maison $(10 \mathrm{~m})$, on considère que la chapelle ne devait compter qu'un seul vaisseau, certainement voûté d'ogives. 


\section{Une chapelle mariale romane?}

8 Comme le faisait déjà remarquer Sandrine Roser : «l'emplacement de cette chapelle au sud-est de l'église, et par conséquent, à l'est de la salle capitulaire - ainsi que son vocable marial laissent penser à une origine bien antérieure à la fondation du $X v^{e}$ siècle ${ }^{12}$. Cette remarque se basait également sur un document du XIV siècle qui laisse soupçonner l'existence d'une chapelle dédiée à la Vierge antérieure à celle de l'abbé Henri de Salins. Une supplique nous apprend en effet qu'en 1381, le frère Joffroy du Vernoy, prêtre et moine à Baume, détenait comme bénéfice «la chapelle NotreDame dans le monastère de Baume ${ }^{13}$. Il s'agissait certainement d'une chapelle horsœuvre, car il semble qu'il n'existait pas d'autre chapelle mariale à l'intérieur de l'abbatiale avant le début du début $\mathrm{du} \mathrm{xv}^{\mathrm{e}}$ siècle, date à laquelle Amé de Chalon (1389-1432) en établit une dans la partie nord du jubé ${ }^{14}$.

9 La chapelle de Salins aurait donc pu ne pas être une construction ex nihilo de l'abbé de Salins au $\mathrm{xv}^{\mathrm{e}}$ siècle, mais résulter d'un agrandissement ou d'un réaménagement d'une chapelle plus ancienne, en partie révélée par l'archéologie du bâti, comme nous allons le voir. En outre, sa situation et son intégration au système de circulation à l'intérieur de l'abbaye tendent à reconnaître dans cet espace architectural une chapelle mariale de type clunisien. Certains actes du début du $\mathrm{Xv}^{\mathrm{e}}$ siècle font état d'un petit cloître au sein du monastère ${ }^{15}$ qui n'est pas localisé, mais dont le seul emplacement pouvant lui correspondre est l'actuelle cour orientale - parfois appelée cour des chanoines ou troisième cour (fig. 1). Ce schéma n'est pas inconnu dans le monde clunisien où un cloître secondaire se développe parfois sur l'un des côtés d'une chapelle dédiée à la Vierge, comme c'est le cas par exemple à Saint-Martin des Champs à Paris. Les prospections géophysiques réalisées en 2010 et 2011 dans l'abbaye révèlent dans la troisième cour des structures qui pourraient appartenir à ce petit cloître, même si nous ne pouvons pas exclure, en l'absence de fouille, que ces traces n'appartiennent pas à des bâtiments antérieurs ou à des réseaux (fig. 3). Quoi qu'il en soit, il n'est plus fait mention du petit cloître baumois après le premier quart $d u x^{e}$ siècle, peut-être en raison des aléas de la recherche documentaire, mais peut-être parce qu'il fut partiellement détruit lors de la construction de la chapelle de Salins. 
Fig. 3 - Carte de la prospection géophysique réalisée dans la cour des chanoines (d'après V. Cachier, université Paris VI-Umr Sisyphe).

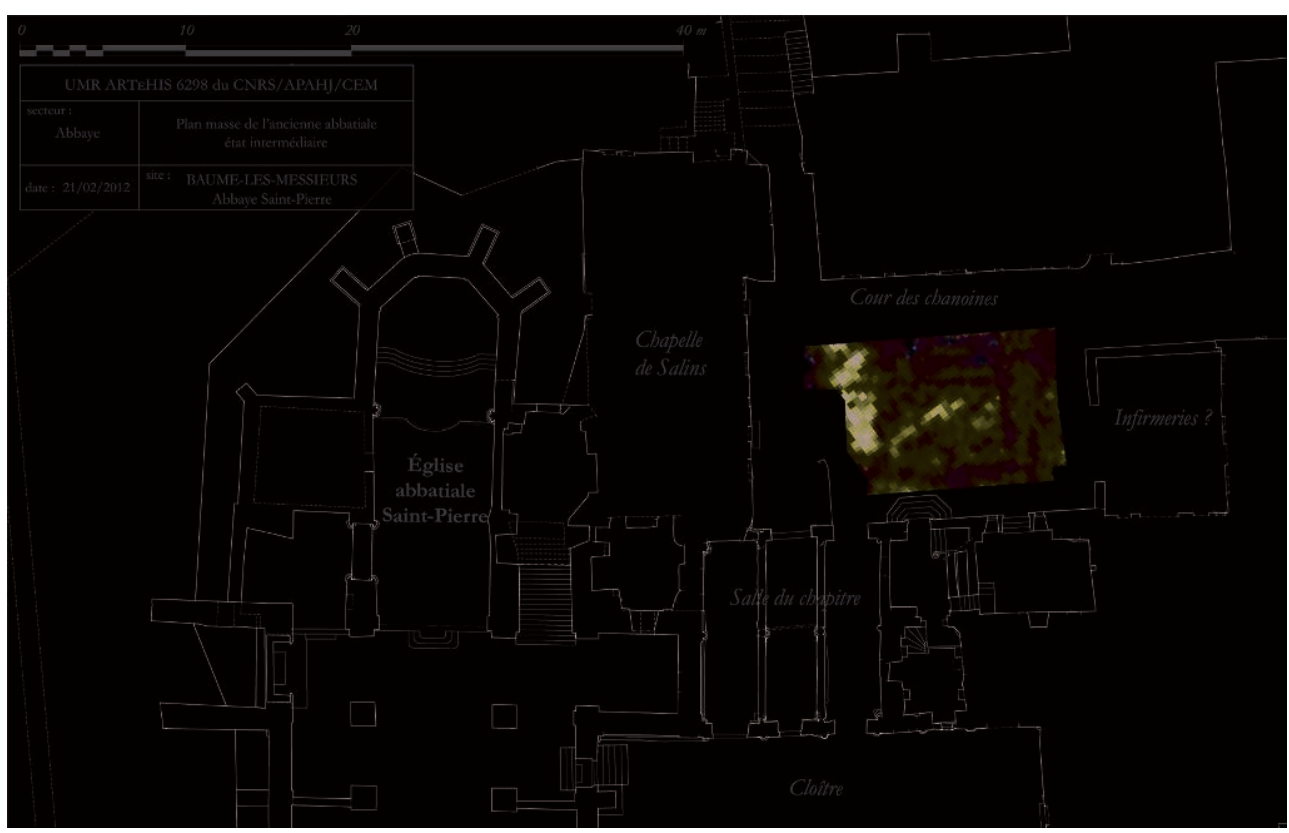

\section{Première analyse archéologique des élévations et essai de datation}

10 Les investigations archéologiques nous ont conduits à prospecter dans la maison aménagée dans l'ancienne chapelle de Salins où une cave voûtée d'arêtes a retenu notre attention en raison de sa situation privilégiée au contact du bras du transept sud de l'église et de l'ancienne salle du chapitre transformée en garage. 
Fig. 4 - Baume-les-Messieurs, vue du mur sud de la « cave Domerc ", première travée de la chapelle (cl. M.-L. Bassi).

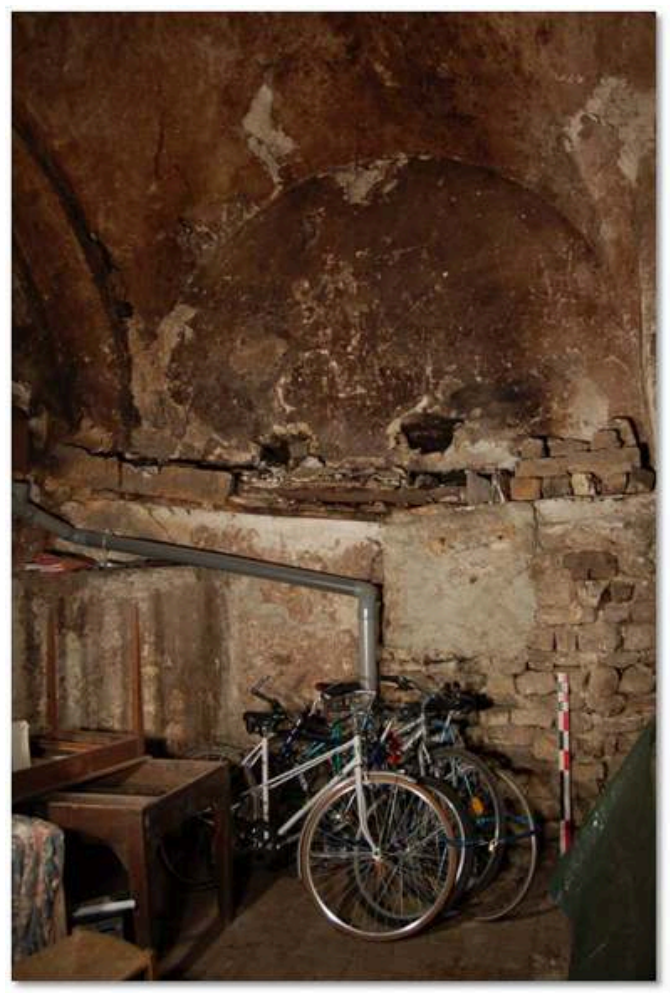

11 Cet espace est identifié dans les textes comme une sacristie à l'époque Moderne, avant de servir de cave à la maison à partir de 1685, au moment où une nouvelle sacristie est créée dans la chapelle au nord de la seconde travée de chœur. On retrouve l'affectation de cet espace comme celle d'une sacristie dans toute l'historiographie baumoise, sous la description d'une salle voûtée d'arêtes.

12 Les sources d'archives ne nous renseignent pas sur la fonction antérieure de cette «cave ", ni à partir de quel moment elle sert de sacristie à l'abbatiale. Mais son emplacement, nos premières observations, sa situation privilégiée avec l'église et la salle capitulaire, ainsi que l'existence présumée d'une chapelle Notre-Dame dès le $\mathrm{XIV}^{\mathrm{e}}$ siècle dans les textes, nous ont laissés supposer que les structures conservées pouvaient appartenir à une chapelle mariale primitive.

13 Les propriétaires actuels nous ont accordé l'autorisation de lever le plan de la cave et de dessiner des coupes (fig. 5) ; en revanche, les nombreuses couches d'enduit limitent la portée de nos observations et interdisent une analyse fine des maçonneries. Il s'agit donc plus d'un état des lieux qu'une véritable étude archéologique du bâti. 
Fig. 5 - Baume-les-Messieurs, relevé en plan et en coupe du secteur de la « cave Domerc » (dessin M.-L. Bassi, d'après équipe de fouille).

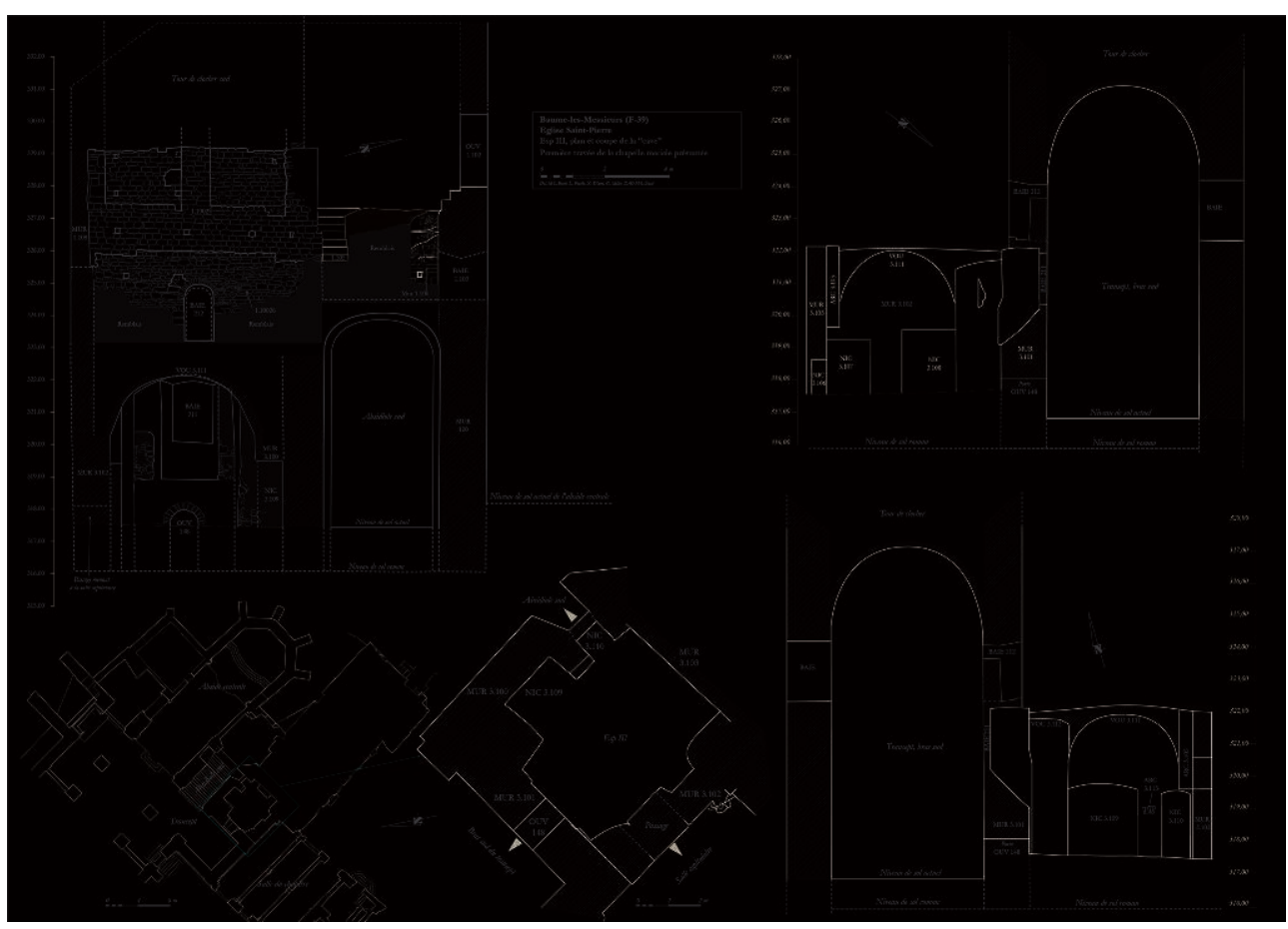

Fig. 6 - Baume-les-Messieurs, détail de l'arc doubleau du mur est (cl. M.-L. Bassi).

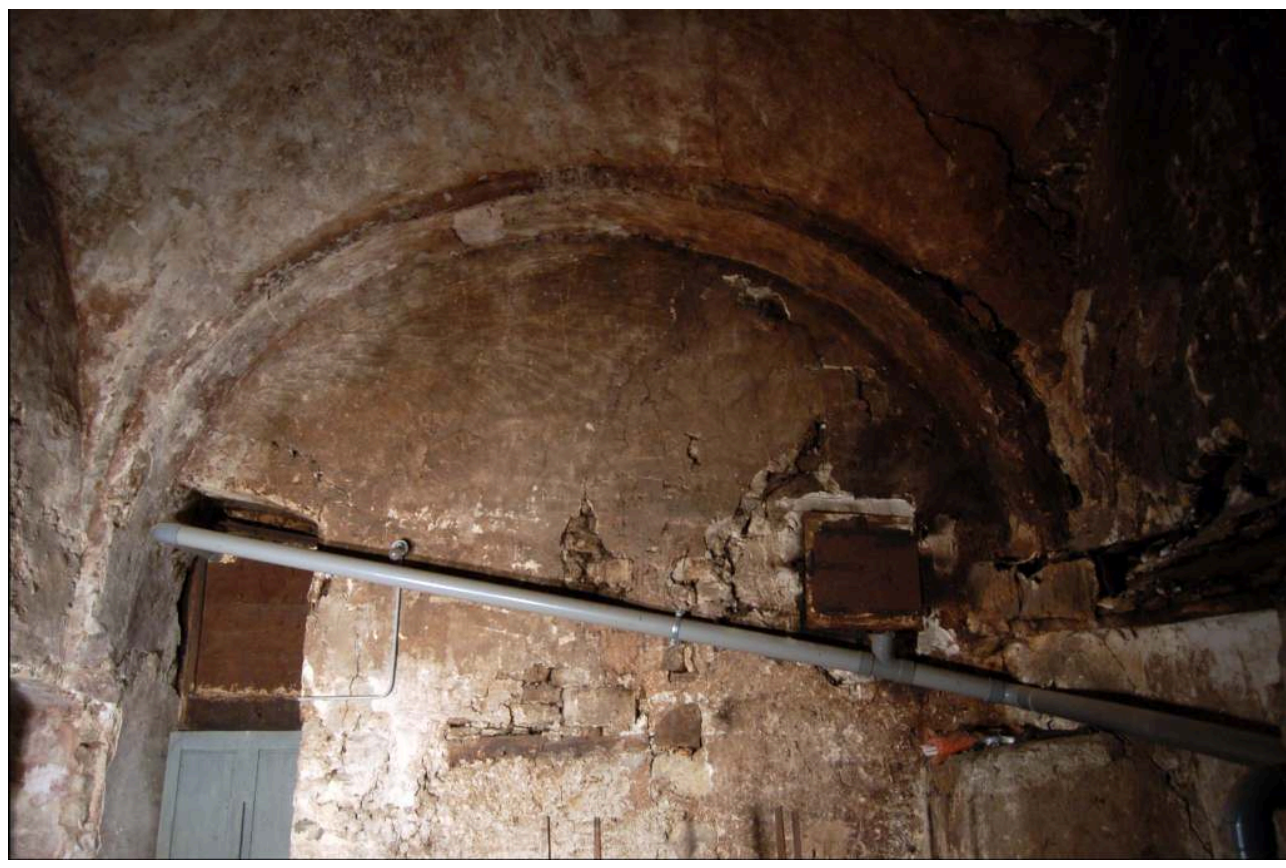

14 La «cave Domerc » s'étend sur une surface d'environ $25 \mathrm{~m}^{2}$ à l'angle que formait l'absidiole sud avec le bras sud du transept. La pièce est couverte d'une voûte d'arêtes limitée à l'est par un arc en plein cintre (fig. 6). Une première analyse a démontré qu'il ne s'agit pas d'un arc formeret marquant la fermeture de cet espace à l'est, mais au contraire, d'un arc doubleau, enchâssé dans une cloison postérieure, autorisant par conséquent la restitution d'une seconde travée dans le prolongement oriental. Le mur 
oriental, construit au niveau de l'arc doubleau est probablement contemporain de la transformation de la travée en sacristie à l'époque Moderne. La travée conservée mesure 4,90 m de longueur pour une largeur de 4,50 m. En ajoutant une seconde travée, on arriverait à une longueur totale de la nef d'environ 10,50 $\mathrm{m}$. La chapelle devait se terminer par une abside semi-circulaire de plus ou moins $2 \mathrm{~m}$ de profondeur si l'on respecte les proportions de la nef, soit une longueur totale d'environ 12,50 $\mathrm{m}$.

Fig. 7 - Baume-les-Messieurs, proposition de restitution du chevet de l'abbatiale et de la chapelle mariale (dessin M.-L. Bassi).

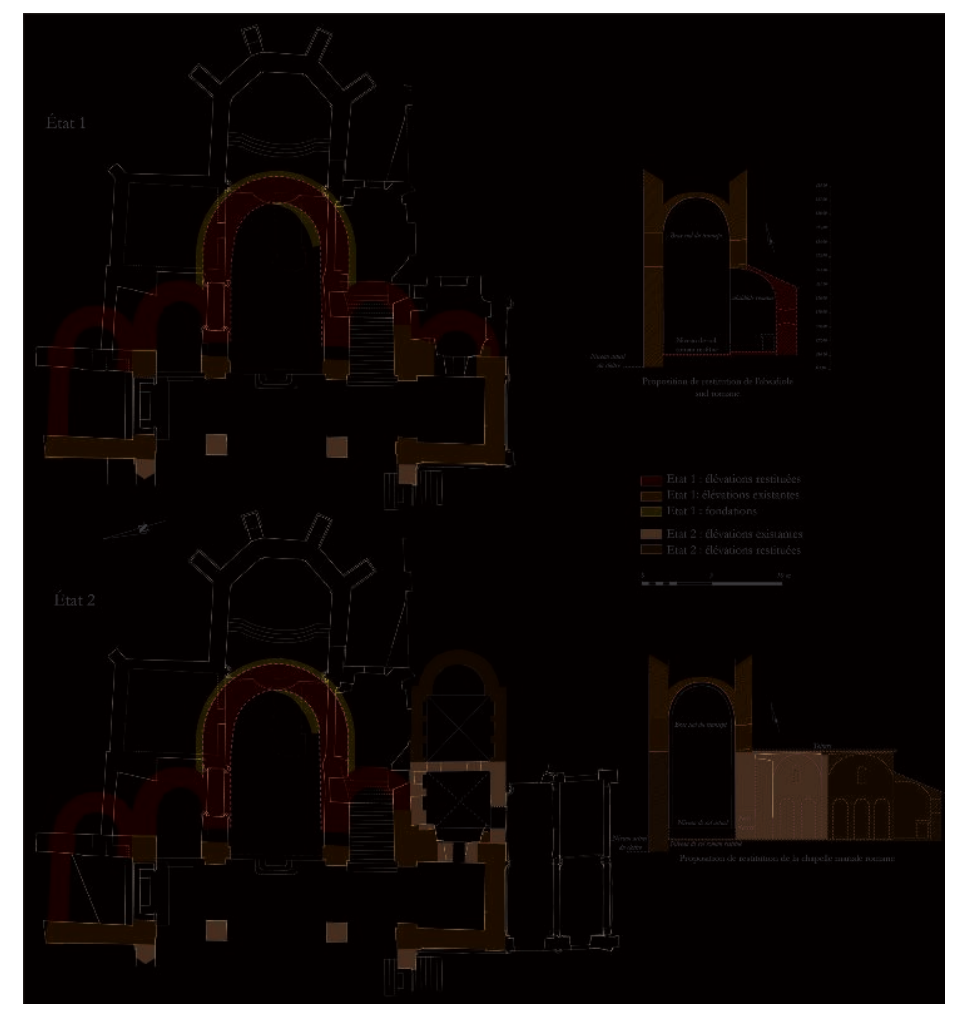

La restitution des parties orientales demeure hypothétique dans l'état actuel de nos connaissances sur le monument, et fait largement appel aux édifices encore conservés ou mieux connus. La situation de la chapelle par rapport au tracé de l'abside centrale romane - telle qu'on le restitue depuis sa découverte dans la fouille du chœur - ne permet pas de la doter d'un transept, comme à Marcigny par exemple, sauf à considérer que la nef était composée de trois travées et non de deux. Mais cette restitution relèverait plus d'une spéculation que de l'interprétation objective des données. Aussi, en resterons-nous à la restitution d'une longueur d'environ 12,50 m pour une largeur de $4,50 \mathrm{~m}$, soit des dimensions légèrement en deçà de celles des chapelles mariales de Charlieu (15 x 5 m), de Marcigny (21,50 x 6 à 7 m) ou encore de Cluny II (15,30 x 11,56 m, d'après Conant). 
Fig. 8 - Baume-les-Messieurs, détail des niches dans le mur nord (cl. S. Bully).

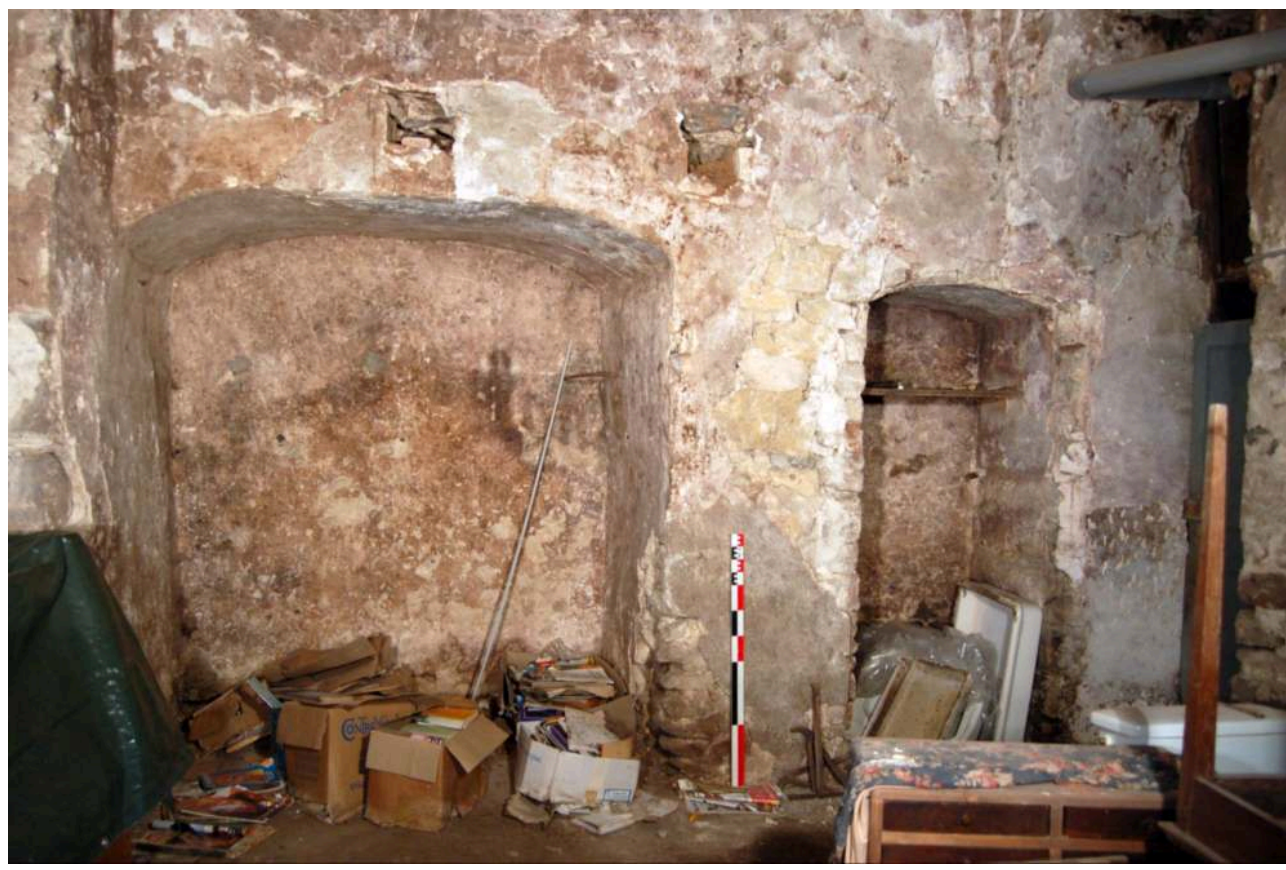

Deux niches maçonnées couvertes d'un arc déprimé sont aménagées dans l'épaisseur du mur gouttereau nord (fig. 8). La niche occidentale est large de 2,14 m pour une profondeur d'environ $1 \mathrm{~m}$ et la niche orientale est large de $0,90 \mathrm{~m}$ et profonde également de $1 \mathrm{~m}$. La niche orientale est aménagée dans une ouverture qui permettait la communication entre cet espace et une chapelle du xiII ${ }^{e}$ siècle prolongeant l'absidiole latérale sud. Ce passage - en partie percé dans le pilastre qui réceptionnait l'arc doubleau roman - est certainement contemporain de l'installation de la sacristie dans la première travée de la chapelle mariale. La grande niche pourrait être contemporaine de la sacristie et destinée au rangement des vêtements sacerdotaux. Mais ses dimensions comme son volume pourraient l'assimiler à un enfeu plus ancien, contemporain, peut-être, de la dernière phase d'utilisation de la chapelle. Cette hypothèse induit également que le niveau de sol actuel serait antérieur à la transformation de la chapelle de Salins en habitation en 1685. Mais surtout, une lacune dans l'enduit entre les deux niches laisse apparaître les vestiges d'un segment d'arc formé de cinq petits moellons calcaires allongés (fig. 5). Cet arc couvre une première niche, profonde de $30 \mathrm{~cm}$, surcreusée sur ses bords par les deux niches tardives. Le tableau oriental de l'ébrasement de la grande niche et le tableau ouest de la seconde sont formés par l'épaisseur du mur d'origine de la chapelle et le bouchage de cette première niche dont le fond, couvert d'un enduit crème, est visible sur une tranche verticale (fig. 9). 
Fig. 9 - Baume-les-Messieurs, détail d'une niche (obturée) de l'hypothétique arcature aveugle du mur nord (cl. S. Bully).

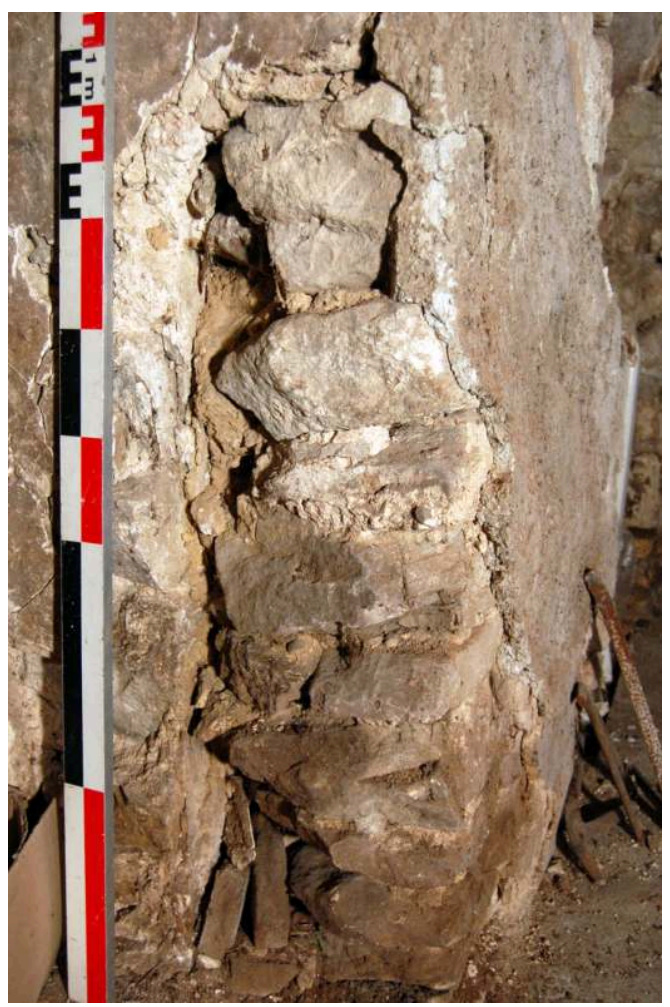

La cote altimétrique de l'arc, comme sa situation en plan, ne permet pas de l'associer au parement externe de l'absidiole sud. Aussi, on émettra l'hypothèse que les parements internes de la chapelle étaient animés par un système d'arcature aveugle dont la niche constituerait un vestige. L'arcature s'élèverait à environ $2,85 \mathrm{~m}$ du sol de la chapelle et serait constituée de cinq arcades par mur si l'on considère que la nef était formée de deux travées (fig. 7).

L'absence d'une analyse détaillée des maçonneries nous oblige à rester prudents sur la datation de la construction, mais les vestiges conservés sont assurément antérieurs à la grande campagne de reconstruction du xiII ${ }^{e}$ siècle qui toucha l'ensemble de l'abbaye. Les reprises du XIII ${ }^{\mathrm{e}}$ siècle se caractérisent toutes par la mise en œuvre de voûtement d'ogives, quelle que soit la monumentalité de la construction. Ici, l'utilisation d'un voûtement d'arêtes avec doubleau en plein cintre indique assurément une construction de l'époque romane. Mais un premier phasage en chronologie relative révèle que la chapelle mariale intervient pourtant dans un second état du chevet de l'abbatiale romane. En effet, la lecture des désordres de maçonneries et la stratigraphie des enduits démontrent que la "cave ", correspondant à la première travée de la nef de la chapelle mariale présumée, succède à une absidiole appartenant au chevet roman primitif de l'abbatiale, composé de cinq absides échelonnées. Les vestiges de l'absidiole semi-circulaire de l'extrémité du transept sont encore visibles dans le mur ouest de la « cave » (fig. 10) où, de part et d'autre d'une large baie moderne, le mur accuse un biais qui paraît être provoqué par des placages de maçonnerie dissimulant les arrachements du départ d'un mur de plan semi-circulaire. 
Fig. 10 - Baume-les-Messieurs, vue du mur ouest avec les traces d'arrachements de l'absidiole primitive (cl. S. Bully).

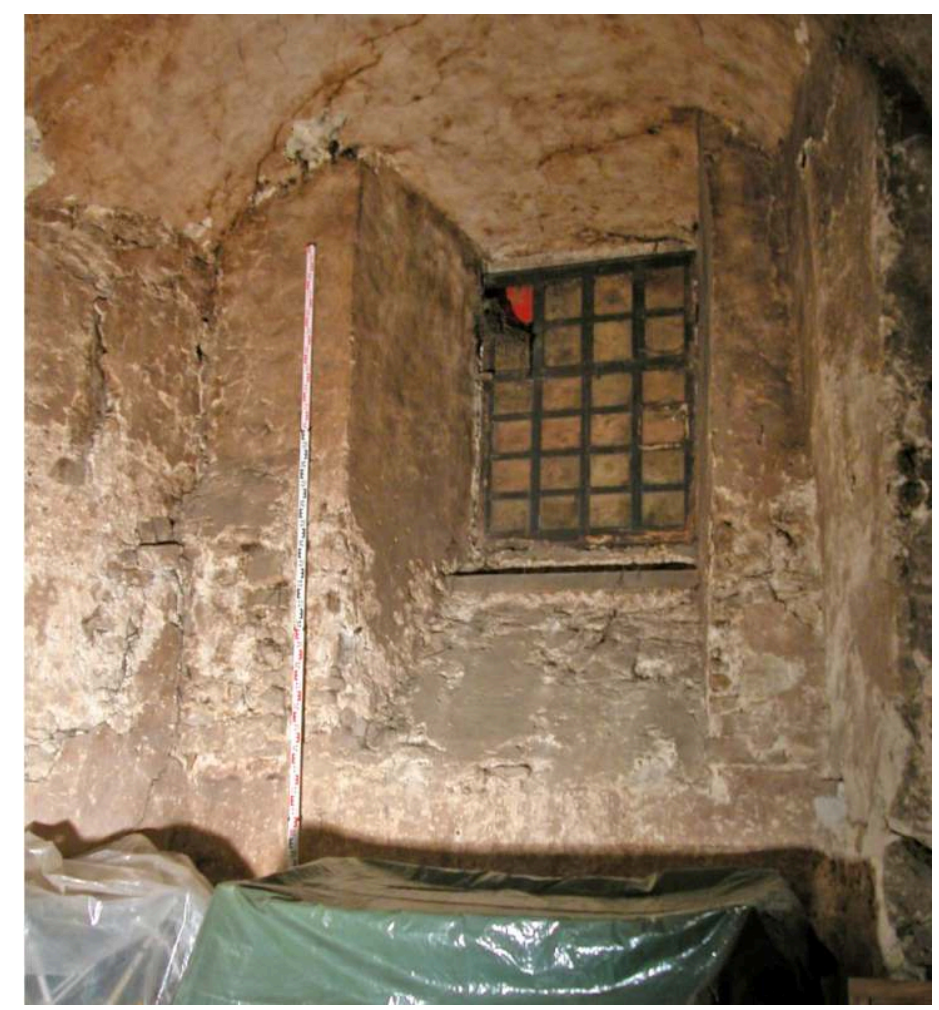

19 En outre, le linteau de la baie moderne présente un abaissement vers l'est de quelques centimètres semblant correspondre aux restes de la voûte en cul-de-four couvrant l'absidiole. C'est lors de la démolition de l'absidiole pour céder la place à la chapelle mariale qu'est nécessairement intervenue la fermeture de son arc d'entrée. La nouvelle chapelle communiquait cependant avec l'église par l'intermédiaire d'une porte aménagée au centre du nouveau mur obturant l'arc d'entrée de l'absidiole détruite. 
Fig. 11 : vue depuis le bras sud du transept de l'ouverture desservant la chapelle mariale (cl. M.L. Bassi).

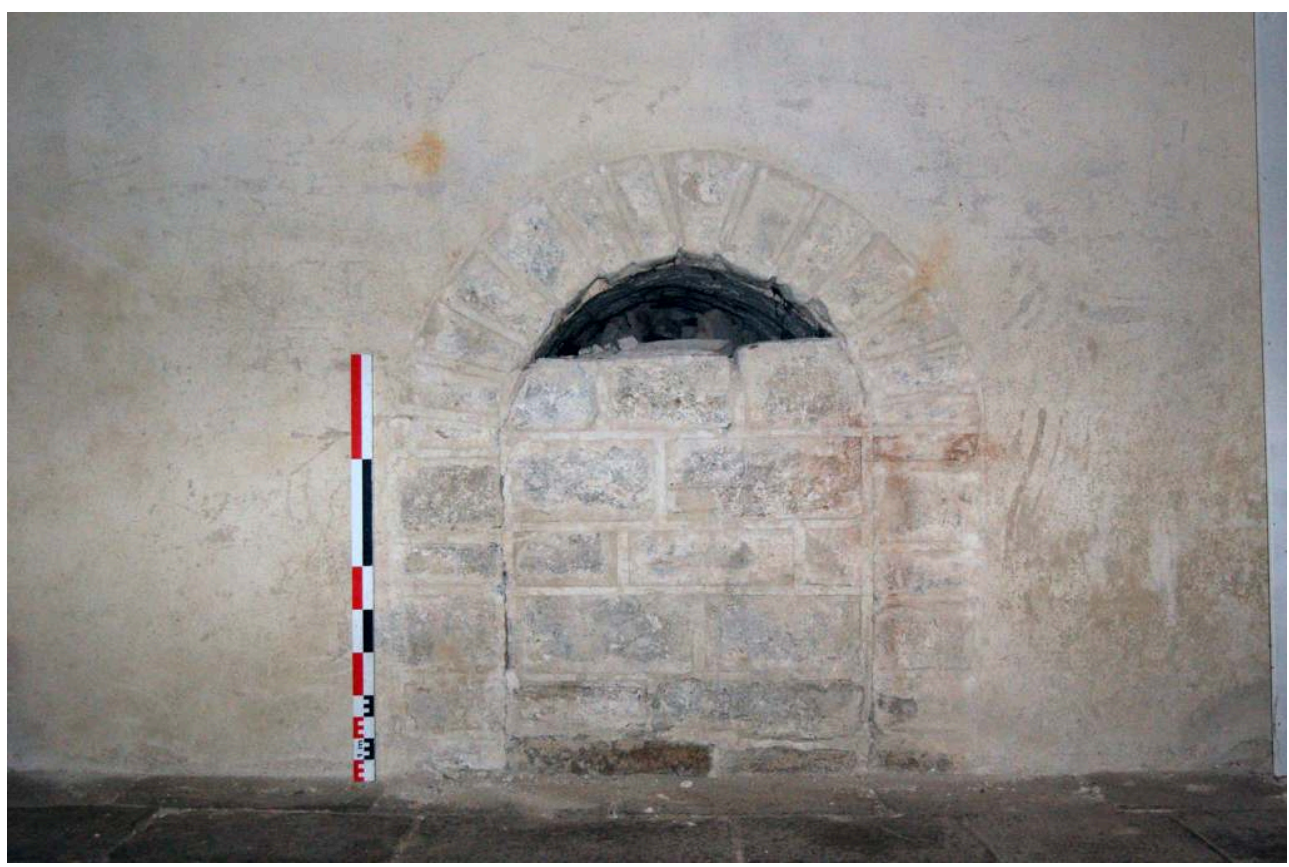

Cette ouverture présente des dimensions modestes, de $0,84 \mathrm{~m}$ de large pour une hauteur visible de $1,25 \mathrm{~m}^{16}$. Son arc en plein cintre et ses piédroits sont constitués de moellons taillés avec soin; la face des blocs calcaire est légèrement bombée avec des angles adoucis. Les pierres qui forment l'arc esquissent la forme de claveaux, liés par un joint de mortier de 1 à $2 \mathrm{~cm}$ d'épaisseur. La taille et le module des pierres sont proches de ce que l'on peut observer sur les piles de la nef qui correspondent à un deuxième état de l'abbatiale où la nef est agrandie et dotée d'une avant-nef ${ }^{17}$ dans la deuxième moitié du XI .

\section{La place de la chapelle dans le cloître}

21 La salle du chapitre du XIII ${ }^{\mathrm{e}}$ siècle est partagée en plusieurs propriétés, réaffectées à divers usages : la travée nord au contact avec la chapelle mariale a été transformée en garage et en chaufferie en partie basse ; une chambre à coucher est aménagée sous ses voûtes. La chapelle communiquait avec le transept à l'ouest, nous l'avons vu, mais également avec la salle du chapitre à travers une seconde ouverture percée dans le mur sud. L'ouverture, murée, est visible depuis l'ancienne salle capitulaire comme depuis la chapelle (cf. fig. 4) : elle mesure 1,50 m de largeur; les enduits dissimulant sa partie supérieure ne permettent pas d'en connaître la hauteur. La différence de niveaux de sols entre la chapelle et la salle du chapitre est estimée à environ $0,80 \mathrm{~m}$, soit près de quatre marches qui devaient prendre place dans l'épaisseur du mur (de 1,43 m). Il est donc vraisemblable que l'on montait dans la chapelle mariale depuis la salle du chapitre à travers une porte à double vantail. L'ampleur de ce passage - par rapport à celui ouvrant sur le transept - témoignerait de l'importance accordée aux déplacements entre la chapelle mariale et le chapitre et du lien « quasi-organique » qui réunissait ces deux entités architecturales, en dépit d'une configuration singulière. On ne sait pas si ce passage est contemporain de la chapelle romane ou s'il a été percé lors de la 
construction ou de la reconstruction du chapitre au xiII siècle Le plan comme la situation de la salle du chapitre antérieure nous est totalement inconnu dans l'attente d'une étude plus poussée des élévations ou d'une fouille de ce secteur. Aussi, s'il est vraisemblable que le "binôme» chapelle mariale/salle du chapitre existait dans le monastère des années 1100 , seule sa configuration du XIII ${ }^{e}$ siècle commence à se dessiner.

L'implantation de la chapelle mariale supposée et son intégration au système de circulation à l'intérieur du monastère correspondent à ce que l'on connait des chapelles mariales clunisiennes, mais avec une légère variante. En effet, elle se situe à l'articulation avec la salle du chapitre, l'église, le cimetière monastique et les infirmeries présumées à travers une cour ou le petit cloître. Mais plus surprenant est sa communication directe avec le bras sud du transept.

Pour la période romane, les circulations entre cette chapelle et les autres " organes" du carré claustral sont délicates. Nous ne possédons aucune donnée concrète sur le cloître roman même s'il est probable que celui $\mathrm{du}_{\mathrm{xv}} \mathrm{v}^{\mathrm{e}}$ en a conservé l'emprise. La salle du chapitre romane est complètement inconnue, tout comme le dortoir qui devait la surmonter. Les ouvertures du bras sud (occuli et baie) du transept semblent exclure un bâtiment à deux étages immédiatement collé au mur sud du transept dans la première moitié du XI ${ }^{\mathrm{e}}$ siècle. Des pierres plates cassées dessinent une ligne horizontale juste sous la baie en plein cintre et peuvent être interprétées comme les vestiges d'un solin ou le faîte d'un appentis. Un passage couvert pouvait donc séparer le bras sud du transept avec la salle capitulaire et le dortoir (fig. 12).

Fig. 12 - Plan général de l'abbaye avec proposition de restitution des circulations (d'après M.-L. Bassi et S. Bully).

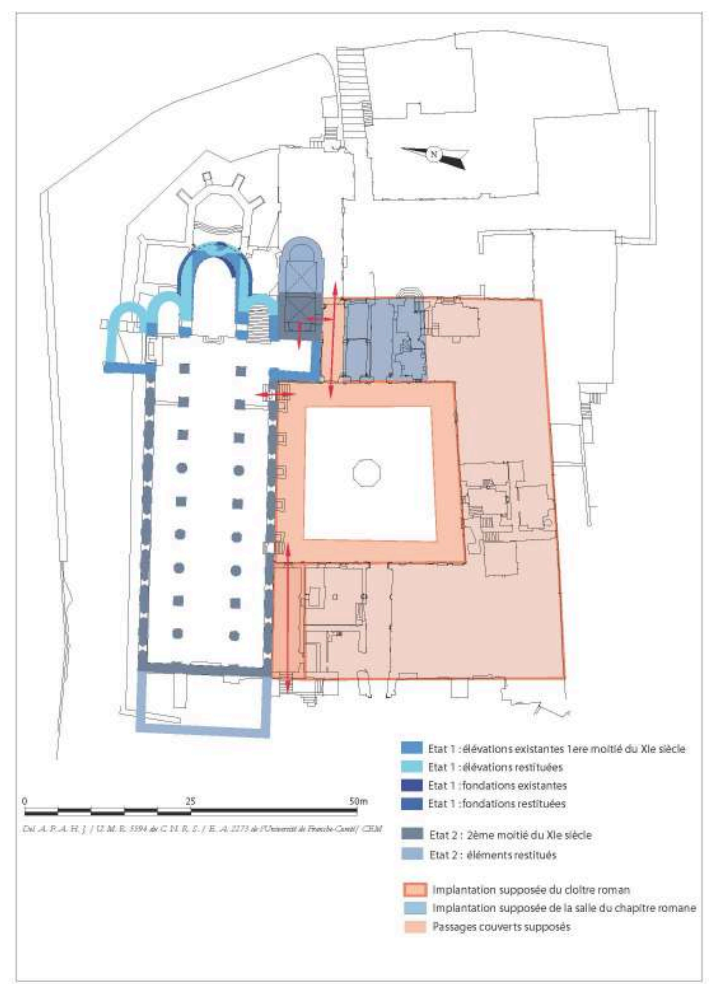



dortoir et la salle du chapitre, pour la période romane, n'a été repéré dans le bâti. La communication entre le chapitre, l'église et la chapelle mariale pouvait se faire par ce passage. Le lien entre la chapelle mariale et l'infirmerie est également inconnu; l'infirmerie à Baume est généralement située dans l'angle sud-ouest de la deuxième cour, mais ses dispositions et son plan restent à déterminer.

\section{Conclusion}

Le croisement des données textuelles avec une première analyse des élévations accrédite l'existence d'une chapelle dédiée à la Vierge dès l'époque romane à l'abbaye de Baume-les-Messieurs. Au XIII ${ }^{\mathrm{e}}$ siècle, la chapelle est assurément au contact de la salle du chapitre alors qu'elle en était peut-être séparée par un couloir dans un état antérieur. Greffée sur le bras sud du transept, elle est également en connexion étroite avec l'église abbatiale. Cette configuration particulière résulte d'un état antérieur. En effet, la chapelle mariale succède à l'absidiole la plus au sud du chevet primitif, en l'amplifiant architecturalement par un développement oriental indépendant. Il n'est donc pas inconcevable de penser que l'absidiole sud accueillait déjà un autel dédié à la Vierge et qu'ainsi, on en ait conservé la titulature, mais tout en redéfinissant un nouvel espace ecclésial avec un plan et des pratiques liturgiques qui lui sont attachés. Sur la base des observations archéologiques - qui demanderaient à être poursuivies et complétées - on entrevoit donc à Baume la traduction, dans les choix architecturaux et dans la partition des espaces, de changement dans la liturgie, peut-être en lien avec le développement du culte marial dans la deuxième moitié $\mathrm{du} \mathrm{XI} \mathrm{e}^{\mathrm{e}}$ siècle.

Il est également intéressant de noter que durant cette période, alors que l'abbaye est encore indépendante, elle va pourtant adopter deux dispositifs clunisiens emblématiques : une avant-nef et une chapelle mariale. Généralement, l'implantation d'une chapelle mariale et d'une galilée dans les prieurés semble intervenir après leur rattachement à Cluny. Leur adoption à Baume est bien antérieure à la soumission de l'abbaye à Cluny en 1147. Si le monastère a adopté ces deux dispositifs, c'est qu'ils répondaient à des besoins liturgiques identiques ou proches de la liturgie clunisienne. Il est difficile de préciser ce constat, mais cela pose de nombreuses questions, notamment sur le lien qui unissait le monastère jurassien à sa fille bourguignonne, entre le départ de l'abbé Bernon en 909 et son intégration à l'Ecclesia cluniacensis 138 ans plus tard.

\section{NOTES}

1. L'église abbatiale Saint-Pierre a fait l'objet de plusieurs campagnes d'étude archéologique de ses élévations, conduites par Marie-Laure Bassi pour sa thèse de doctorat intitulée : L'abbatiale de Baume-les-Messieurs à l'époque romane : histoire d'un chantier, université de Franche-Comté, 2013. Cette étude a été réalisée dans le cadre du programme de recherche de l'université de Franche-Comté sur «L'architecture des églises comtoises au Moyen Âge à travers l'archéologie

Bulletin du centre d'études médiévales d'Auxerre | BUCEMA, Hors-série nº 6 | 2013 
du bâti » dirigé par É. Vergnolle et S. Bully. L'abside centrale romane a été grande partie découverte en 2011 lors de la fouille programmée du chœur dirigé par les auteurs de ces lignes.

2. Nous remercions sincèrement la famille Domerc-Navarre de nous avoir autorisé l'accès à leur cave à plusieurs reprises.

3. ADJ, 1 F 25 : Notice sur l'abbaye de Baume (vers 1760), p. 47.

4. D. MONNIER, « Baume » in Annuaire du Jura, Lons-le-Saunier, 1843, p. 58.

5. A. ROUSSET, Dictionnaire géographique, historique et statistique des communes de la FrancheComté et des hameaux qui en dépendent, classé par département. Département du Jura, Besançon, 1853, t. I, p. 171.

6. L.-A. RoY, L'abbaye de Baume-les-Messieurs, Baume-les-Messieurs, 1928, p. 28.

7. R. LOCATELli, P. GResSeR, R. FIETIER, G. MOYSE et J. COURTIEU, L'abbaye de Baume-les-Messieurs, Lonsle-Saunier, 1978, p. 190.

8. S. ROSER, L'art à l'abbaye de Baume-les-Messieurs dans la première moitie du $X V^{e}$ siècle, Thèse de doctorat, sous la direction d'É. Vergnolle, Besançon, 2003.

9. ADJ, 1 H 7 - Inventaire des titres (1754), p. 17-18.

10. ADJ, 1 H 21.

11. ADJ, 1 F 14 - Traité entre l'abbé et les religieux (13/06/1685).

12. S. ROSER, L'art à l'abbaye..., p. 592

13. Ce renseignement, communiqué à Sandrine Roser par M. Gérard Moyse, directeur des archives de la Côte-d'Or, a été trouvé dans le registre des suppliques $n^{\circ} 61$, conservé aux Archives du Vatican (fo $48 \mathrm{v}^{\circ}$; visée par la chancellerie pontificale le 05/03/1381) ; S. ROSER, L'art à l'abbaye..., p. 593.

14. Ibid., p. 593

15. Bibliothèque municipale de Besançon, collection Dunand, ms. 30 (XVIII ${ }^{\mathrm{e}} \mathrm{s}$ ), p. 182.

16. Le rehaussement du niveau de sol dans le bras du transept comme dans la cave a tronqué toute la partie basse du passage.

17. Une avant-nef a été identifiée à la suite d'une prospection géophysique et d'un sondage en 2011.

\section{INDEX}

Index géographique : France/Baume-les-Messieurs

Mots-clés : monastère, archéologie du bâti, abbatiale romane, chevet à absides échelonnées, chapelle funéraire, chapelle mariale, salle du chapitre, cloître, circulation

\section{AUTEURS}

\section{SÉBASTIEN BULLY}

CNRS, ARTEHIS-UMR6298

MARIE-LAURE BASSI

Université de Franche-Comté 